\section{P071 AUTOANTIBODIES AGAINST SERUM AMYLOID A REDUCE IL-6 RELEASE FROM PERIPHERAL BLOOD MONONUCLEAR CELLS}

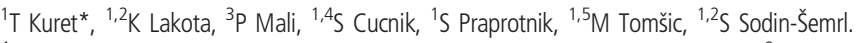
${ }^{1}$ Department of Rheumatology, University Medical Centre Ljubljana, Ljubljana; ${ }^{2} F A M N I T$, University of Primorska, Koper; ${ }^{3}$ Blood Transfusion Centre; ${ }^{4}$ Faculty of Pharmacy; ${ }^{5}$ Faculty of Medicine, University of Ljubljana, Ljubljana, Slovenia

\subsection{6/annrheumdis-2018-EWRR2018.88}

Introduction Serum amyloid A (SAA) is a sensitive inflammatory marker rapidly increased during the acute phase, followed by a steady decline to physiological levels during resolution. While resolution and SAA reduction have been documented, the exact mechanism remains elusive. Although antibodies against SAA (anti-SAA) have been previously identified in healthy blood donors (HBDs) in smaller, preliminary studies, their potential function is still unclear. ${ }^{1,2}$

Objectives To detect anti-SAA and anti-SAA $1 \alpha$ in the sera of 300 HBDs using ELISA, characterise their subclasses and avidity. Additionally, we aimed to evaluate their presence in intravenous immunoglobulin (IVIG) and potential effects on released IL-6 from SAA-treated peripheral blood mononuclear cells (PBMCs).

Methods An in-house ELISA was adapted from Rosenau and Schur $^{1}$ and developed ${ }^{2}$ for detection of anti-SAA and antiSAA1 $\alpha$. Both antibody fractions were isolated from IVIG using MicroLink Protein Coupling Kit (Thermo Scientific). PBMCs were purified from $5 \mathrm{HBDs}$ by density gradient centrifugation and stimulated with SAA or SAA1 $\alpha(1.5 \mu \mathrm{g} / \mathrm{ml})$ in the presence/absence of anti-SAA and anti-SAA $1 \alpha$ for 5 hours, $37^{\circ} \mathrm{C}$. IL-6 concentration was measured in supernatants by ELISA (Invitrogen).

Results The median (IQR) absorbance in HBDs was 0.655 (0.262-1.293) for anti-SAA and $0.493(0.284-0.713)$ for antiSAA $1 \alpha$. Both anti-SAA and anti-SAA1 $\alpha$ reached peak levels between 41-50 years and diminished with age, with women exhibiting significantly higher levels than men. Good positive correlation was observed between anti-SAA and anti-SAA1 $\alpha$. Both antibodies were prevalently of the IgG subclass, with heterogeneous to high avidity and were detected also in IVIG. Stimulation of PBMCs with SAA significantly induced IL-6 release (mean \pm SD) $(389.5 \pm 184.4 \mathrm{pg} / \mathrm{ml})$ with levels decreasing significantly upon addition of $4.5(131.4 \pm 44.4 \mathrm{pg} / \mathrm{ml})$ or $9.0 \mu \mathrm{g} / \mathrm{ml}(118.1 \pm 57.4 \mathrm{pg} / \mathrm{ml})$ anti-SAA. A similar trend was also found for SAA1 $\alpha$ and anti-SAA $1 \alpha$.

Conclusions Anti-SAA could play a physiological role in downregulating proinflammatory activity of SAA and could represent an attractive, novel therapeutic option for patients with chronic inflammatory diseases.

\section{REFERENCES}

1. Rosenau BJ, Schur PH. Antibody to serum amyloid A. I Autoimmun 2004:23:179-82.

2. Lakota K, Thallinger GG, Cucnik S, Bozic B, Mrak-Poljsak K, Ambrozic A, et al. Could antibodies against serum amyloid a function as physiological regulators in humans? Autoimmunity 2011;44:149-58.

Acknowledgements The authors would like to acknowledge funding from the Slovenian Research Agency (ARRS) for the National Research Program P3-0314.

Disclosure of interest None declared

\section{P072 IRF1 IS CRITICAL FOR THE INFLAMMATORY GENE EXPRESSION IN FIBROBLAST-LIKE SYNOVIOCYTES}

${ }^{1} \mathrm{M}$ Bonelli*, ${ }^{1} \mathrm{~S}$ Hayer, ${ }^{1} \mathrm{~K}$ Dalwigk, ${ }^{1} \mathrm{~B}$ Niederreiter, ${ }^{2} \mathrm{~T}$ Pap, ${ }^{1} \mathrm{~J}$ Smolen, ${ }^{1} \mathrm{H}$ Kiener,

${ }^{1} \mathrm{~T}$ Karonitsch. 'Devision of Rheumatology, Medical University of Vienna, Vienna, Austria;

${ }^{2}$ Institute of Experimental Musculoskeletal Medicine, Muenster, Germany

\subsection{6/annrheumdis-2018-EWRR2018.89}

Introduction Fibroblast-like synoviocytes (FLS) are increasingly recognised as major pathogenic cells in synovial inflammation of patients with Rheumatoid Arthritis (RA). In response to pro-inflammatory stimuli, such as TNF, FLS produce vast amounts of cytokines and chemokines that help to recruit and activate immune cells and drive the local inflammatory process. The pathways and transcription factors that determine the inflammatory response in FLS are, however, largely unexplored.

Objectives To investigated the potential contribution of the transcription-factor IRF1 to the inflammatory gene expression in FLS.

Methods Expression of IRF1 in synovial tissue samples (12 RA and 8 osteoarthritis (OA) patients) was assessed by immunohistochemistry (IHC). Moreover, FLS were isolated according to established protocols and cultured using 2-D or 3-D culture techniques. IRF1expression in response to TNF was determined by western blots, qPCR, immunofluorescence microscopy or IHC. FLS were also stimulated with TNF in the presence or absence of IRF1 siRNA pools. Expression of pro-inflammatory cytokines and chemokines was measured by qPCR.

Results IRF1 expression was significantly increased in rheumatoid synovial tissues as compared to patients with OA on protein level. RA-FLS stimulation with TNF ex vivo caused rapid upregulation of IRF1 and proved the involvement of TNF in the regulation of IRF1. Immunofluorescence analysis further revealed that IRF1 was mainly localised in the nucleus of TNF-stimulated FLS. Moreover, also chronic TNF exposure of FLS grown in a 3-D synovial tissue culture system promoted the expression of IRF1. siRNA-mediated knockdown of IRF1 in FLS significantly reduced the TNF-induced expression of pro-inflammatory cytokines and chemokines, such as IL6, CCL7, CXCL11 and TNFSF13B, which confirmed the role of IRF1 as a critical regulator of proinflammatory genes in RA FLS.

Conclusions Our data reveal that IRF1 is crucial for the inflammatory response of FLS and support the idea that IRF1 might be an exciting therapeutic target in patients with RA.

Disclosure of interest None declared

\section{P073 REGULATION OF JOINT DESTRUCTION BY ACTIVIN A IN RHEUMATOID ARTHRITIS}

V Kracke*, J Intemann, M Fennen, T Pap, B Dankbar. Institute of Musculoskeletal Medicine, Münster, Germany

\subsection{6/annrheumdis-2018-EWRR2018.90}

Introduction Activins and inhibins belong to the transforming growth factor $\beta$ family. Activins are disulphide-linked homoor heterodimers consisting of two inhibin $\beta$ chains $(\beta A, \beta B)$ that are expressed in many cell types. However, activin A ( $\beta A$ $\beta A$ ) is the only activin that is expressed in bone and cartilage. Moreover, activin A has been demonstrated not only to stimulate receptor activator of $\mathrm{NF}-\kappa \mathrm{B}$ ligand (RANKL)-induced 
osteoclast (OC) differentiation but also to inhibit osteoblast differentiation.

Objectives Here we investigate the impact of activin a on joint destruction in rheumatoid arthritis.

Methods Synovial tissue samples from rheumatoid arthritis (RA) and osteoarthritis (OA) patients were analysed by immunohistochemical staining. For in vitro experiments, bone marrow-derived macrophages (BMM) were isolated from femurs and tibias of WT mice and differentiated into osteoclasts in the presence of macrophage colony-stimulating factor (M-CSF) and RANKL with or without activin A. OC differentiation was characterised by TRAP staining. Resorption activity was determined by quantification of osteoclast-mediated pit formation on a calcium phosphate-coated plate. Proliferation of BMMs was evaluated using the CyQUANT Cell Proliferation Assay Kit. Furthermore, osteoclast-specific gene expression as well as the activation of SMAD2, MAPK and NF-kB signalling were analysed by immunoblotting. The interaction of phospho-SMAD2 with NFATc1 was evaluated by co-immunoprecipitation using Dynabeads.

Results We demonstrate that activin A is highly abundant in the synovium of RA but not of OA patients. In vitro, activin A strongly enhanced the RANKL-mediated differentiation of BMMs into mature OCs, reflected by a significantly increased OC number, OC size and number of nuclei per OC compared to the conventional treatment with RANKL alone. Moreover, concomitant administration of activin A led to a significant increase of the total resorption area as well as resorption area per pit, indicating an increased activity of individual OCs. Effects of activin A on BMMs were not caused by increased proliferation since no effect on M-CSF-driven proliferation was observed. Furthermore, activin A alone was not able to induce the expression of OC differentiation markers, but the RANKL-induced expression was enhanced by activin A. After stimulation with activin A, BMMs showed an activation of SMAD2, but not of MAPK p38, ERK, JNK or NF-kB. Finally, co-stimulation of RANKL and activin A resulted in an increased interaction of activated SMAD2 with NFATc1.

Conclusions The data strongly suggest that increased expression of activin $\mathrm{A}$ in the arthritic joint is most likely associated with enhanced osteoclast formation, promoting joint destruction in rheumatoid arthritis.

Disclosure of interest None declared

\section{P074 IL-10 REGULATES SKIN THICKNESS AND SCALING IN IMIQUIMOD-INDUCED PSORIASIS-LIKE SKIN INFLAMMATION IN MICE}

${ }^{1} \mathrm{X} X \mathrm{U}^{*},{ }^{1} \mathrm{E}$ Prens, ${ }^{1} \mathrm{E}$ Florencia, ${ }^{2} \mathrm{~L}$ Boon, ${ }^{1} \mathrm{P}$ Asmawidjaja, ${ }^{1} \mathrm{~A}-\mathrm{M}$ Otten-Mus, ${ }^{1} \mathrm{E}$ Lubberts. ${ }^{1}$ Erasmus MC Rotterdam, Rotterdam; ${ }^{2}$ Bioceros, Utrecht, Netherlands

\subsection{6/annrheumdis-2018-EWRR2018.91}

Introduction Psoriasis is an autoimmune skin disease affecting around $0.6 \%$ to $3 \%$ of the whole population with detrimental physical and societal impacts. Previously, we established a psoriasis-like skin inflammation model in mice using topical application of imiquimod (IMQ). This model successfully recaptures all critical features of clinical psoriasis such as keratinocyte hyperproliferation, munro's microabscesses, and shares a similar infiltration profile of various immune cells. Previous data suggest up-regulation of IL-10, but its role in this psoriasiform model is not clear.
Objectives To investigate the role of IL-10 in the IMQinduced psoriasis-like skin inflammation.

Methods Psoriasis-like skin inflammation was induced by topical application of imiquimod (Aldara) for 5 or 10 days. Mice were injected intraperitoneally with anti-IL-10 or an isotype control antibody or subcutaneously with dexamethasone. Back skin of mice was scored for up to 10 days using a modified Psoriasis Area and Severity Index (PASI) score system adapted from clinical PASI score. Inflammation and skin thickness were scored histologically. Gene expression and immune cells in the skin were analysed using RT-PCR and flow cytometry, respectively.

Results At day 10, both skin thickness and scaling score were significantly higher after neutralising IL-10 compared to isotype control, or either group compared to dexamethasonetreated animals. At days 5 and $10, \mathrm{H}$ and $\mathrm{E}$ staining confirmed that epidermal thickness was more prominent in antiIL-10 treated mice compared to isotype control or dexamethasone-treated mice, with more profound differences at day 10 . Ki-67 staining for proliferating keratinocytes showed more proliferation at the epidermal basal layer after neutralising IL10. In addition, significant more infiltration of neutrophils was found in skin at day 10. At day 5, IL-23/IL-17 pathway cytokines were more significantly upregulated in anti-IL-10 group than the isotype control group, while at day 10, a significant upregulation of IL-19, IL-24 expression were found in anti-IL-10 group compared to isotype control.

Conclusions IL-10 regulates skin thickness and scaling during psoriasis-like skin inflammation. Furthermore, our data suggested that IL-10 might influence psoriatic symptoms through dampening of IL-23/IL-17 axis in early phase (day 5) and reducing IL-19 and IL-24 expression at late stage (day 10). The negative feedback signal of IL-10 partially explains the observed decrease of inflammation in imiquimod-induced skin inflammation after day 5 .

Disclosure of interest X. Xu: None declared, E. Prens: None declared, E. Florencia: None declared, L. Boon: None declared, P. Asmawidjaja: None declared, A.-M. Otten-Mus: None declared, E. Lubberts Grant/research support from: Novartis

\section{P075 CHANGES OF METABOLIC BIOMARKER LEVELS UPON ANTI-TNF THERAPY IN RHEUMATOID ARTHRITIS}

${ }^{1}$ A Pusztai ${ }^{*},{ }^{1} E$ Végh, ${ }^{1} A$ Horváth, 'S Szántó, 'G Szucs, ${ }^{1}$ A Hamar, ${ }^{1}$ A Domján, ${ }^{1} K$ Hodosi, ${ }^{2}$ Seres, ${ }^{3} \mathrm{G}$ Kerekes, ${ }^{1} \mathrm{Z}$ Szekanecz. ${ }^{1}$ Division of Rheumatology, Department of Medicine; ${ }^{2}$ Division of Metabolic Disease, Department of Medicine; ${ }^{3}$ Division of Intensive Care Unit, Department of Medicine, University of Debrecen, Faculty of Medicine, Debrecen, Hungary

\subsection{6/annrheumdis-2018-EWRR2018.92}

Introduction Rheumatoid arthritis (RA) has been associated with cardiovascular disease and metabolic syndrome. Numerous pro-inflammatory cytokines (e.g. TNF- $\alpha$, IL-1, IL-6) are released, which cytokines cause increased reactive oxygen species (ROS) production and thereby contribute to the increased lipid peroxidation and reduction of many antioxidants. These processes not only lead to the deterioration of joints and other tissues but may also contribute to comorbidities, such as atherosclerosis.

Objectives The aim of this study was to assess the effects of anti-TNF therapy on different metabolic markers, such as PON1 (paraoxonase 1), arylesterase, chemerin and 